Internat. J. Math. \& Math. Sci.

Vol. 23, No. 11 (2000) 795-799

S0161171200002817

(c) Hindawi Publishing Corp.

\title{
MHD FLOW OF AN ELASTICO-VISCOUS FLUID UNDER PERIODIC BODY ACCELERATION
}

\author{
E. F. EL-SHEHAWEY, ELSAYED M. E. ELBARBARY, \\ N. A. S. AFIFI, and MOSTAFA ELSHAHED
}

(Received 19 February 1999)

\begin{abstract}
Magnetohydrodynamic (MHD) flow of blood has been studied under the influence of body acceleration. With the help of Laplace and finite Hankel transforms, an exact solution is obtained for the unsteady flow of blood as an electrical conducting, incompressible and elastico-viscous fluid in the presence of a magnetic field acting along the radius of the pipe. Analytical expressions for axial velocity, fluid acceleration and flow rate has been obtained.
\end{abstract}

Keywords and phrases. Blood flow, integral transforms, body acceleration, magnetic field. 2000 Mathematics Subject Classification. Primary 76Z05.

1. Introduction. In situations like travel in vehicles, aircraft, operating jackhammer and sudden movements of body during sports activities, the human body experiences external body acceleration. Prolonged exposure of a healthy human body to external acceleration may cause serious health problem like headache, increase in pulse rate and loss of vision on account of disturbances in blood flow Majhi and Nair [3].

It has been established that the biological systems in general are greatly affected by the application of external magnetic field. So far, the theoretical studies dealing with the influence of applied magnetic field on blood flow have received very little attention Ramachandra Rao and Deshikachar [4].

Many researchers have studied blood flow in the artery by considering blood as either Newtonian or non-Newtonian fluids, since blood is a suspension of red cells in plasma; it behaves as a non-Newtonian fluid at low shear rate. Chaturani and Palanisamy [1] studied pulsatile flow of blood through a rigid tube under the influence of body acceleration as a Newtonian fluid. In the present work, we consider the unsteady flow of blood as an elastico-viscous magnetohydrodynamic fluid in a circular pipe. It is assumed that a magnetic field along the radius of the pipe is present, no external electric field is imposed and magnetic Reynolds number is very small. The main idea of our work is the mathematical study of these phenomena in order to obtain analytical expression for the axial velocity, flow rate, fluid acceleration and shear stress.

2. Mathematical formulation. Consider the motion of blood as an electrically conducting, incompressible and non-Newtonian fluid in the presence of a magnetic field acting along the radius of a circular pipe. We assume that the magnetic Reynolds 
number of the flow is taken to be small enough, so that the induced magnetic and electric field can be neglected. We consider the flow as axially symmetric, pulsatile and fully developed. The pressure gradient and body acceleration $G$ are given by:

$$
\begin{aligned}
-\frac{\partial p}{\partial z} & =A_{0}+A_{1} \cos (\omega t), \quad & t \geq 0, \\
G & =a_{0} \cos \left(\omega_{1} t+\phi\right), \quad & t \geq 0,
\end{aligned}
$$

where $A_{0}$ is the steady-state part of the pressure gradient, $A_{1}$ is the amplitude of the oscillatory part, $\omega=2 \pi f$ and $f$ is heart pulse frequency, $a_{0}$ is the amplitude of body acceleration, $\omega_{1}=2 \pi f_{1}$ and $f_{1}$ is body acceleration frequency, $\phi$ is its phase difference, $z$ is the axial distance, and $t$ is time.

Under the above mentioned assumption, the equation of motion for flow as discussed by Stephanie and Rowland [2] in cylindrical polar coordinates can be written in the form:

$$
\rho \frac{\partial u}{\partial t}=A_{0}+A_{1} \cos \omega t+a_{0} \cos \left(\omega_{1} t+\phi\right)+\left(\mu+\mu_{1} \frac{\partial}{\partial t}\right)\left(\frac{\partial^{2} u}{\partial r^{2}}+\frac{1}{r} \frac{\partial}{\partial r}\right)-\sigma B_{0}^{2} u,
$$

where $u(r, t)$ is velocity in the axial direction, $\rho$ and $\mu$ are the density and viscosity of blood, $\mu_{1}$ is the elastico-viscosity coefficient of the fluid, $\sigma$ is the electrical conductivity, $B_{0}$ is the external magnetic field and $r$ is the radial coordinate.

Let us introduce the following dimensionless quantities:

$$
\begin{aligned}
u^{*}=\frac{u}{\omega R}, & r^{*}=\frac{r}{R}, \quad t^{*}=t \omega, & A_{0}^{*}=\frac{R}{\mu \omega} A_{0}, \\
A_{1}^{*}=\frac{R}{\mu \omega} A_{1}, & a_{0}^{*}=\frac{R}{\mu \omega} a_{0}, & z^{*}=\frac{z}{R} .
\end{aligned}
$$

In terms of these variables, equation (2.3) [if dropping the stars] becomes

$$
\alpha^{2} \frac{\partial u}{\partial t}=A_{0}+A_{1} \cos (t)+a_{0} \cos (b t+\phi)+\left(1+\beta \frac{\partial}{\partial t}\right)\left(\frac{\partial^{2} u}{\partial r^{2}}+\frac{1}{r} \frac{\partial u}{\partial r}\right)-H^{2} u,
$$

where $\beta=\left(\omega \mu_{1} / R \mu\right)$ is dimensionless parameter governing elastico-viscosity of the fluid. $\alpha=R(\omega p / \mu)^{1 / 2}$ is (Womersley parameter), $H=B_{0} R(\sigma / \mu)^{1 / 2}$, is the (Hartmann number), $b=\left(\omega_{1} / \omega\right)$ and $R$ is the radius of the pipe.

We assume that at $t<0$, only the pumping action of the heart is present and at $t=0$, the flow in the artery corresponds to the instantaneous pressure gradient, i.e., $-\partial p / \partial z=A_{0}+A_{1}$. As a result, the flow velocity at $t=0$ is given by:

$$
u(r, 0)=\frac{A_{0}+A_{1}}{H^{2}}\left(1-\frac{I_{0}(H r)}{I_{0}(H)}\right),
$$

where $I_{0}$ is a modified Bessel function of first kind of order zero, when $H$ tends to zero, we obtain the velocity of the classical Hagen-Pioseuille flow.

$$
u(r, 0)=\frac{A_{0}+A_{1}}{4}\left(1-r^{2}\right) .
$$

The initial and boundary conditions for our problem are:

$$
u(r, 0)=\frac{A_{0}+A_{1}}{H^{2}}\left(1-\frac{I_{0}(H r)}{I_{0}(H)}\right),
$$




$$
\begin{aligned}
& u(1, t)=0, \\
& u(0, t) \text { is finite. }
\end{aligned}
$$

3. Required integral transforms. If $f(r)$ satisfies Dirichlet conditions in closed interval $(0,1)$ and if its finite Hankel transform Senddon [5, page 82$]$ is defined to be:

$$
f^{*}\left(\lambda_{n}\right)=\int_{0}^{1} r f(r) J_{0}\left(r \lambda_{n}\right) d r
$$

where $\lambda_{n}$ are the roots of the equation $J_{0}(r)=0$. Then at each point of the interval at which $f(r)$ is continuous:

$$
f(r)=2 \sum_{n=1}^{\infty} f^{*}\left(\lambda_{n}\right) \frac{J_{0}\left(r \lambda_{n}\right)}{J_{1}^{2}\left(\lambda_{n}\right)},
$$

where the sum is taken over all positive roots of $J_{0}(r)=0, J_{0}$ and $J_{1}$ are Bessel function of first kind.

The Laplace transform of any function is defined as:

$$
\bar{f}(s)=\int_{0}^{\infty} e^{-s t} f(t) d t, \quad R s>0 .
$$

4. Analysis. Employing the Laplace transforms (3.3) to equation (2.5) in the light of (2.8a) we get:

$$
\begin{aligned}
\alpha^{2} s \bar{u}-\alpha^{2} u(r, 0)= & \frac{A_{0}}{s}+\frac{A_{1} s}{s^{2}+1}+\frac{a_{0}(s \cos \phi-b \sin \phi)}{s^{2}+b^{2}} \\
& +\left(\frac{\partial^{2}}{\partial r^{2}}+\frac{1}{r} \frac{\partial}{\partial r}\right)[\bar{u}+\beta s \bar{u}-\beta u(r, 0)]-H^{2} \bar{u},
\end{aligned}
$$

where

$$
\bar{u}(r, s)=\int_{0}^{\infty} e^{-s t} u(r, t) d t
$$

Now applying the finite Hankel transforms (3.1) to(4.1) and using (2.8b) we obtain:

$$
\begin{aligned}
\bar{u}^{*}\left(\lambda_{n}, s\right)=\frac{J_{1}\left(\lambda_{n}\right)}{\lambda_{n}}\{ & \frac{A_{0}}{\lambda_{n}^{2}+H^{2}}\left(\frac{1}{s}-\frac{1}{s+h}\right) \\
& +\frac{A_{1}\left(\lambda_{n}^{2}+H^{2}\right)}{\left(\lambda_{n}^{2}+H^{2}\right)^{2}+\left(\alpha^{2}+\lambda_{n}^{2} \beta\right)^{2}}\left[\frac{-1}{s+h}+\frac{s}{s^{2}+1}+\frac{\alpha^{2}+\lambda_{n}^{2} \beta}{\left(\lambda_{n}^{2}+H^{2}\right)\left(s^{2}+1\right)}\right] \\
& +\frac{a_{0}\left(\lambda_{n}^{2}+H^{2}\right) \cos \phi}{\left(\lambda_{n}^{2}+H^{2}\right)^{2}+\left(\alpha^{2}+\lambda^{2} \beta\right) b^{2}}\left[\frac{-1}{s+h}+\frac{s}{s^{2}+b^{2}}+\frac{\left(\alpha^{2}+\lambda_{n}^{2} \beta\right) b^{2}}{\left(s^{2} b^{2}\right)\left(\lambda_{n}^{2}+H^{2}\right)}\right] \\
& -\frac{a_{0} b \sin \phi\left(\alpha^{2}+\lambda_{n}^{2} \beta\right)^{2}}{\left(\lambda_{n}^{2}+H^{2}\right)^{2}+b^{2}\left(\alpha^{2}+\lambda_{n}^{2} \beta\right)^{2}}\left[\frac{1}{s+h}-\frac{s}{s^{2}+b^{2}}+\frac{\lambda_{n}^{2}+H^{2}}{\left(s^{2}+b^{2}\right)\left(\alpha^{2}+\lambda_{n}^{2} \beta\right)}\right] \\
& \left.+\frac{A_{0}+A_{1}}{\left(\lambda_{n}^{2}+H^{2}\right)} \frac{1}{(s+h)}\right\},
\end{aligned}
$$

where

$$
h=\frac{\lambda_{n}^{2}+H^{2}}{\alpha^{2}+\lambda_{n}^{2} \beta},
$$


Now the Laplace and finite Hankel inversion of equation (4.3) gives the final solution as:

$$
\begin{aligned}
& u(r, t)=2 \sum_{n=1}^{\infty} \frac{J_{0}\left(\lambda_{n} r\right)}{\lambda_{n} J_{1}\left(\lambda_{n}\right)}\left\{\frac{A_{0}}{\lambda_{n}^{2}+H^{2}}+\frac{A_{1}\left[\left(\lambda_{n}^{2}+H^{2}\right) \cos t+\left(\alpha^{2}+\beta \lambda_{n}^{2}\right) \sin t\right]}{\left(\lambda_{n}^{2}+H^{2}\right)^{2}+\left(\alpha^{2}+\lambda^{2} \beta\right)^{2}}\right. \\
& +\frac{a_{0}\left[\left(\lambda_{n}^{2}+H^{2}\right) \cos (b t+\phi)+\left(\alpha^{2}+\beta \lambda_{n}^{2}\right) \sin (b t+\phi)\right]}{\left(\lambda_{n}^{2}+H^{2}\right)^{2}+b^{2}\left(\alpha^{2}+\beta \lambda_{n}^{2}\right)^{2}} \\
& +e^{-h t}\left[\frac{A_{0}}{\lambda_{n}^{2}+H^{2}}+\frac{A_{1}\left(\lambda_{n}^{2}+H^{2}\right)}{\left(\lambda_{n}^{2}+H^{2}\right)^{2}+\left(\alpha^{2}+\beta \lambda_{n}^{2}\right)^{2}}-\frac{A_{0}+A_{1}}{\lambda^{2}+H^{2}}\right. \\
& \left.\left.+\frac{a_{0}\left[\left(\lambda_{n}^{2}+H^{2}\right) \cos \phi+\left(\alpha^{2}+\lambda_{n}^{2} \beta\right) \sin \phi\right]}{\left(\lambda_{n}^{2}+H^{2}\right)^{2}+b^{2}\left(\alpha^{2}+\lambda_{n}^{2} \beta\right)^{2}}\right]\right\} \text {. }
\end{aligned}
$$

When $\beta$ and $H$ tends to zero, then our solution given by (4.5) reduces to the case considered by Chaturani and Palanisawy [1].

The expression for the flow rate $Q$ can be written as:

$$
Q=2 \int_{0}^{1} r u d r
$$

then

$$
\begin{array}{r}
Q(r, t)=4 \sum_{n=1}^{\infty} \frac{1}{\lambda_{n}^{2}}\left\{\frac{A_{0}}{\lambda_{n}^{2}+H^{2}}+\frac{A_{1}\left[\left(\lambda_{n}^{2}+H^{2}\right) \cos t+\left(\alpha^{2}+\beta \lambda_{n}^{2}\right) \sin t\right]}{\left(\lambda^{2}+H^{2}\right)^{2}+\left(\alpha^{2}+\lambda^{2} \beta\right)^{2}}\right. \\
+\frac{a_{0}\left[\left(\lambda^{2}+H^{2}\right) \cos (b t+\phi)+\left(\alpha^{2}+\beta \lambda_{n}^{2}\right) \sin (b t+\phi)\right]}{\left(\lambda^{2}+H^{2}\right)^{2}+b^{2}\left(\alpha^{2}+\beta \lambda_{n}^{2}\right)^{2}} \\
+e^{-h t}\left[\frac{A_{0}}{\lambda^{2}+H^{2}}+\frac{A_{1}\left(\lambda_{n}^{2}+H^{2}\right)}{\left(\lambda^{2}+H^{2}\right)^{2}+\left(\alpha^{2}+\beta \lambda_{n}^{2}\right)^{2}}-\frac{A_{0}+A_{1}}{\lambda^{2}+H^{2}}\right. \\
\left.\left.+\frac{a_{0}\left[\left(\lambda_{n}^{2}+H^{2}\right) \cos \phi+\left(\alpha^{2}+\lambda_{n}^{2} \beta\right) \sin \phi\right]}{\left(\lambda_{n}^{2}+H^{2}\right)^{2}+b^{2}\left(\alpha^{2}+\lambda_{n}^{2} \beta\right)^{2}}\right]\right\} .
\end{array}
$$

Similarly the expression for fluid acceleration $F$ can be obtained from:

$$
F(r, t)=\frac{\partial u}{\partial t}
$$

Then we have

$$
\begin{aligned}
& F(r, t)=2 \sum_{n=1}^{\infty} \frac{J_{0}\left(\lambda_{n} r\right)}{\lambda_{n} J_{1}\left(\lambda_{n}\right)}\left\{\frac{A_{0}}{\lambda_{n}^{2}+H^{2}}-\frac{A_{1}\left[\left(\lambda_{n}^{2}+H^{2}\right) \sin t-\left(\alpha^{2}+\beta \lambda_{n}^{2}\right) \cos t\right]}{\left(\lambda_{n}^{2}+H^{2}\right)^{2}+\left(\alpha^{2}+\lambda^{2} \beta\right)^{2}}\right. \\
& -\frac{a_{0} b\left[\left(\lambda_{n}^{2}+H^{2}\right) \sin (b t+\phi)-\left(\alpha^{2}+\beta \lambda_{n}^{2}\right) \cos (b t+\phi)\right]}{\left(\lambda_{n}^{2}+H^{2}\right)^{2}+b^{2}\left(\alpha^{2}+\beta \lambda_{n}^{2}\right)^{2}} \\
& -\frac{1}{h} e^{-h t}\left[\frac{A_{0}}{\lambda^{2}+H^{2}}+\frac{A_{1}\left(\lambda_{n}^{2}+H^{2}\right)}{\left(\lambda^{2}+H^{2}\right)^{2}+\left(\alpha^{2}+\beta \lambda_{n}^{2}\right)^{2}}-\frac{A_{0}+A_{1}}{\lambda^{2}+H^{2}}\right. \\
& \left.\left.+\frac{a_{0}\left[\left(\lambda_{n}^{2}+H^{2}\right) \cos \phi+\left(\alpha^{2}+\lambda_{n}^{2} \beta\right) \sin \phi\right]}{\left(\lambda_{n}^{2}+H^{2}\right)^{2}+b^{2}\left(\alpha^{2}+\lambda_{n}^{2} \beta\right)^{2}}\right]\right\} \text {. }
\end{aligned}
$$




\section{REFERENCES}

[1] P. Chaturani and V. Palanisamy, Pulsatile flow of blood with periodic body acceleration, Int. J. Eng. Sci. 29 (1991), no. 1, 113-121. Zbl 825.76983.

[2] S. A. Gilligan and R. S. Jones, Unsteady flow of an elastico-viscous fluid past a circular cylinder, Z. Angew. Math. Phys. 21 (1970), 786-797. Zbl 217.24802.

[3] S. N. Majhi and V. R. Nair, Pulsatile flow of third grade fluids under body accelerationmodeling blood flow, Int. J. Eng. Sci. 32 (1994), no. 5, 839-846. Zbl 925.76975.

[4] A. Ramachandra Rao and K. S. Deshikachar, MHD oscillatory flow of blood through channels of variable cross section, Int. J. Eng. Sci. 24 (1986), 1615-1628. Zbl 625.76129.

[5] I. N. Sneddon, Fourier Transforms, McGraw-Hill Book Co., Inc., New York, Toronto, London, 1951. MR 13,29h. Zbl 038.26801.

El-Shehawey, Elbarbary, Afifi, ANd Elshahed: Department of Mathematics, Faculty of Education, Ain Shams University, Roxy, Heliopolis, CaIro, Egypt 


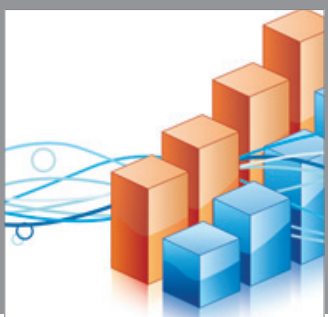

Advances in

Operations Research

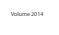

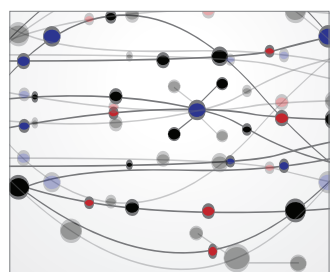

\section{The Scientific} World Journal
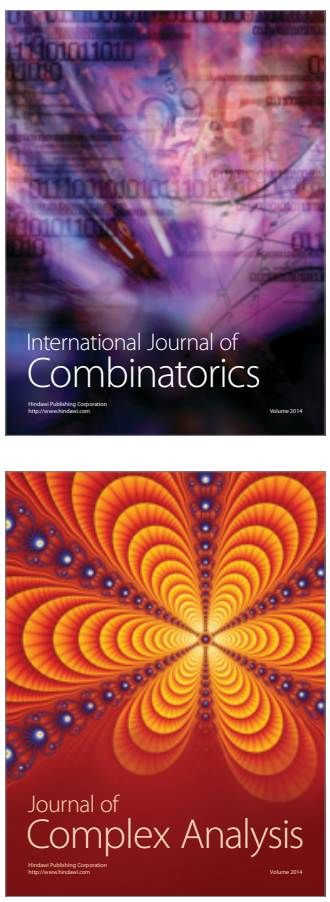

International Journal of

Mathematics and

Mathematical

Sciences
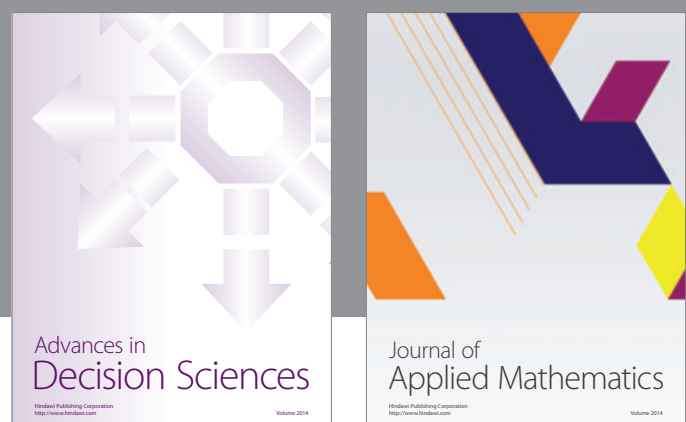

Journal of

Applied Mathematics
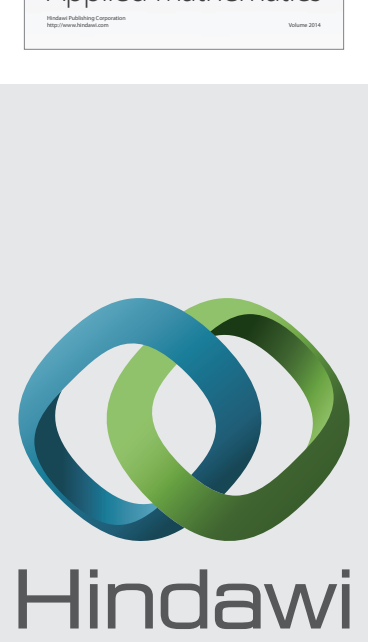

Submit your manuscripts at http://www.hindawi.com
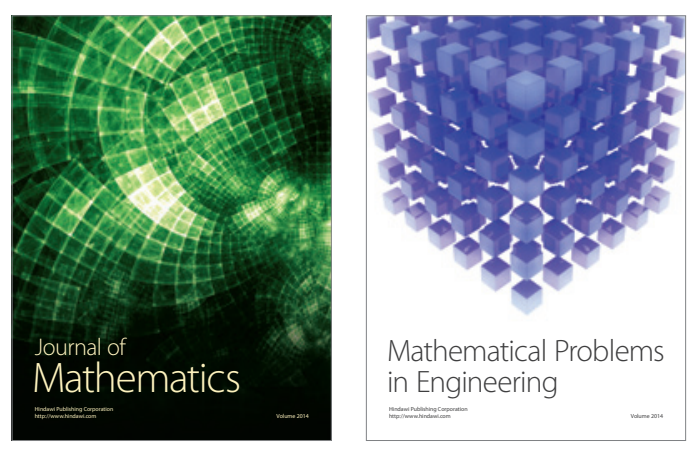

Mathematical Problems in Engineering
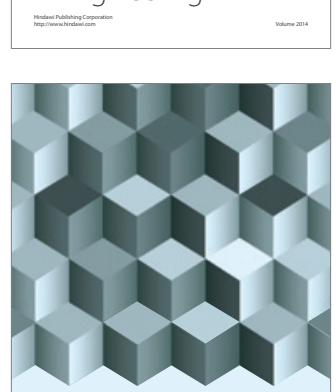

Journal of

Function Spaces
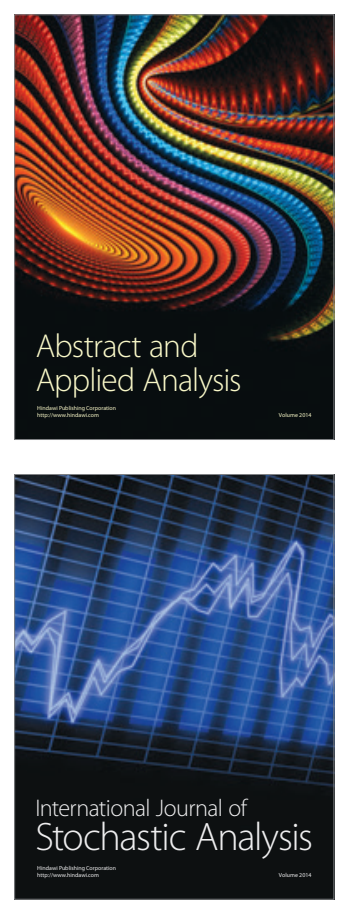

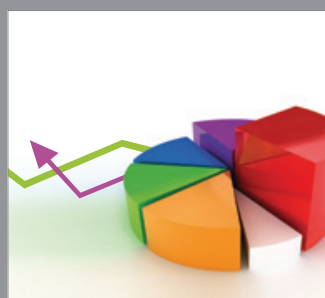

ournal of

Probability and Statistics

Promensencen
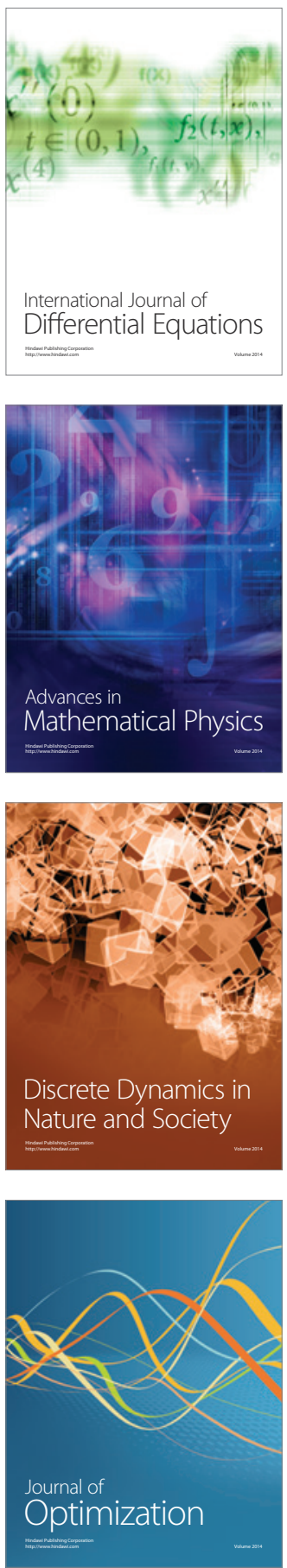